\title{
Anterolateral approach for mini-open rotator cuff repair
}

\author{
Chul-Hyun Cho • Kwang-Soon Song • \\ Byung-Woo Min • Gu-Hee Jung • Young-Kuk Lee • \\ Hong-Kwan Sin
}

Received: 14 May 2011 / Accepted: 8 June 2011 / Published online: 30 June 2011

(C) Springer-Verlag 2011

\begin{abstract}
Purpose This study was undertaken to introduce an anterolateral approach for mini-open rotator cuff repair and evaluate its clinical outcome and effectiveness.

Methods We evaluated 128 consecutive cases that were repaired by mini-open repair using an anterolateral approach. There were 80 men and 48 women, with an average age of 56.2 years. Average follow-up was 25.7 months. There were eight partial-thickness, 26 small, 40 medium, 39 large and 15 massive tears. After arthroscopic glenohumeral examination and subacromial decompression, we made a 3 - to 4-cm skin incision from anterolateral edge of the acromion and dissected to the raphe between the anterior and middle deltoid. The torn tendon was repaired with single- or doublerow technique using suture anchors. To prevent avulsion of the deltoid from the acromion, additional suturing within the bone tunnel was performed. We retrospectively evaluated clinical outcomes using the American Shoulder and Elbow Surgeon (ASES) scoring system.
\end{abstract}

Katharine O'Moore-Klopf, ELS, of East Setauket, New York, USA, provided English-language copyediting.

C.-H. Cho $(\bowtie) \cdot$ K.-S. Song $\cdot$ B.-W. Min

Department of Orthopedic Surgery, Pain Research Center,

Dongsan Medical Center, School of Medicine,

Keimyung University,

194 Dongsan-dong, Joong-gu,

Daegu, Korea 700-712

e-mail: oscho5362@dsmc.or.kr

G.-H. Jung

Department of Orthopedic Surgery, Gospel Hospital,

School of Medicine, Kosin University,

Busan, Korea

Y.-K. Lee $\cdot$ H.-K. Sin

Department of Orthopedic Surgery, Hanmi Hospital,

Daegu, Korea
Results The average visual analogue scale (VAS), activity of daily living (ADL) and ASES scores improved, respectively, from $6.6,12.0$ and 36.7 preoperatively to 1.2, 26.6 and 88.2 postoperatively. There were 71 excellent, 39 good, ten fair and eight poor results. There were no statistically significant difference between final ASES scores and age, symptom duration, tear size or preoperative stiffness, but men had significantly higher final ASES scores than women $(P=0.014)$.

Conclusion Anterolateral approach for mini-open rotator cuff repair produces satisfactory results. It may also provide better visualisation for rotator cuff tears of all sizes.

\section{Introduction}

With advances in shoulder arthroscopy, repair techniques for rotator cuff tear have evolved from open techniques to arthroscopically assisted mini-open techniques and then to an all-arthroscopic technique [1-4]. Recently, arthroscopic rotator cuff repair has been more popular, and satisfactory outcomes were reported by many authors [1, 5-7]. Its potential advantages include less postoperative pain, an extremely low deltoid morbidity and faster rehabilitation. Despite these advantages, this procedure is technically demanding and requires a great deal of practice for a surgeon to obtain proficiency $[1,5,8]$. Because of the technical demands of this procedure, many surgeons still consider the mini-open technique to be the gold standard for rotator cuff repair [3, 4, 6]. Satisfactory clinical outcomes for mini-open technique have been well documented and compared favourably with those for open or arthroscopic repair technique [1, 2, 5, 7-16].

Mini-open repair with a lateral deltoid-splitting approach is commonly used and generally produces good long-term 
results comparable with those seen in many open-repair series $[10,11,14,15,18]$. However, attempts to repair a large or massive tear can still lead to significant deltoid and axillary-nerve injury from excessive traction. Assessment of medially retracted tendons or subscapularis tears is also difficult. The anterior approach provides good visualisation and avoids axillary nerve injury, whereas assessment of posterior cuff tears by the anterior approach is difficult [11, 19, 20]. Matsen and Lippitt [21] described mini-open repair using a deltoid-on approach through a $3-$ to $4-\mathrm{cm}$ split in the most prominent anterolateral raphe of the deltoid muscle near its origin at the acromion.

In an attempt to better assess rotator cuff tears, we used a modified deltoid-on approach. Our technique represents a smaller deltoid-sparing version of standard open repair, preserving the deltoid origin by splitting the anterolateral raphe of the deltoid muscle and still allowing adequate exposure for rotator cuff repair. Thus, the purpose of this study was to introduce the anterolateral approach for miniopen rotator cuff repair and evaluate its clinical outcome and effectiveness.

\section{Materials and methods}

Between August 2006 and December 2008, 159 consecutive patients with rotator cuff tear underwent mini-open repair by a single surgeon using an anterolateral approach. Of those patients, 128 followed up for over one year were evaluated. We obtained approval from our institutional review board for our study. Average patient age was 56.2 (range 39-74) years, and there were 80 men and 48 women, among which 90 (70.3\%) were dominant and 38 (29.7\%) were nondominant limbs. The average duration of symptoms before surgery was 28.1 (range one month-20) years, and the average follow-up period was 25.7 (range 12-45) months. Of the 128 patients, $18(14.1 \%)$ had preoperative shoulder stiffness.

\section{Surgical technique}

With patients placed in the lateral decubitus position and given general anesthaesia, we performed a standard arthroscopic glenohumeral examination to evaluate intraarticular pathology through the posterior and anterior portals. The entire rotator cuff was assessed, and the tear was defined with respect to size and location. The arthroscope was then placed in the subacromial space through the posterior portal, and a lateral portal was created approximately 3-4 cm distal from the lateral border of the acromion. After resecting the hypertrophic bursa and performing tenolysis of the retracted tendon using a full-radius shaver and a radiofrequency ablation device, we performed arthroscopic subacromial decompression through a lateral portal using an acromionizer burr. We evaluated the size and location of the torn tendon and carefully débrided the tear margin and greater tuberosity. The tear was repaired by mini-open technique using an anterolateral approach. A 3- to 4-cm skin incision was made from the anterolateral edge of the acromion distally, and dissection was made to the raphe between the anterior and middle deltoid (Fig. 1). A stay suture was placed distally to prevent propagation of the deltoid split and potential injury to the axillary nerve. After assessing the adequacy of the acromioplasty by direct digital palpation, we placed a deltoid retractor for direct visualisation of the rotator cuff and humeral head (Fig. 2). As the torn tendon was tagged by traction sutures after removing the hypertrophic bursal tissue around the split site to improve visualisation, we confirmed involvement and configuration of the torn tendon by rotating the arm and attempted anatomical reduction on the footprint of the greater tuberosity. After preparing the footprint using a ring curette or rasp, the torn tendon was repaired by single- or doublerow technique using suture anchors (Fig. 3). If pathology of the long head of the biceps tendon was found, tenodesis was performed under direct visualisation. To prevent avulsion of the deltoid from the acromion, additional suturing within the bone tunnel was performed using no. 1 absorbable sutures with cutting needle (Ethicon, Cornelia, GA, USA) (Fig. 4).

Wearing an abduction brace, patients began pendulum and passive range-of-motion exercises one day after surgery. They began active range-of-motion exercises six weeks after surgery, muscle-strengthening exercises at three months and occupational or sports activities at six months.

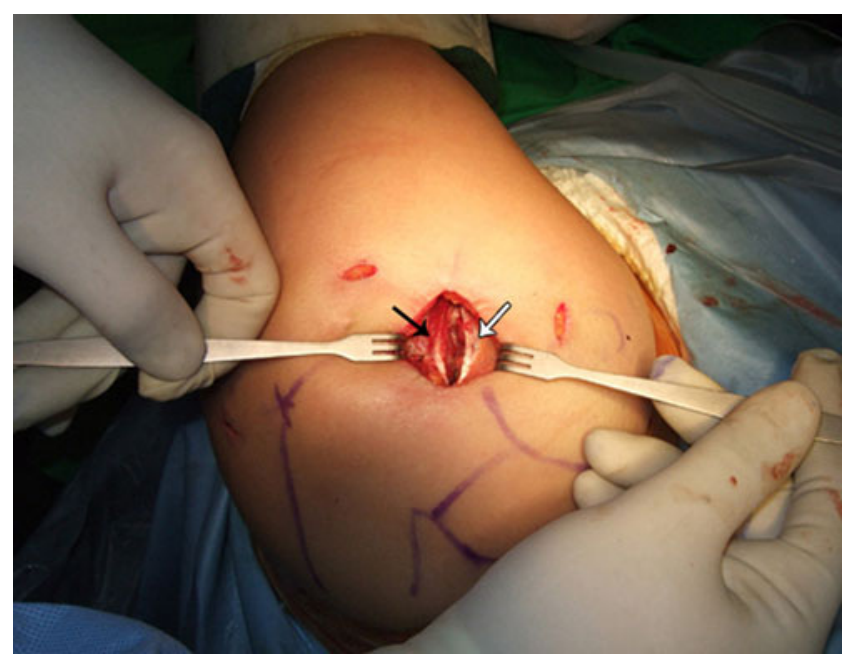

Fig. 1 A 3- to 4-cm skin incision was made distally from the anterolateral edge of the acromion, with dissection to the raphe between the anterior (white arrow) and middle (black arrow) deltoid 


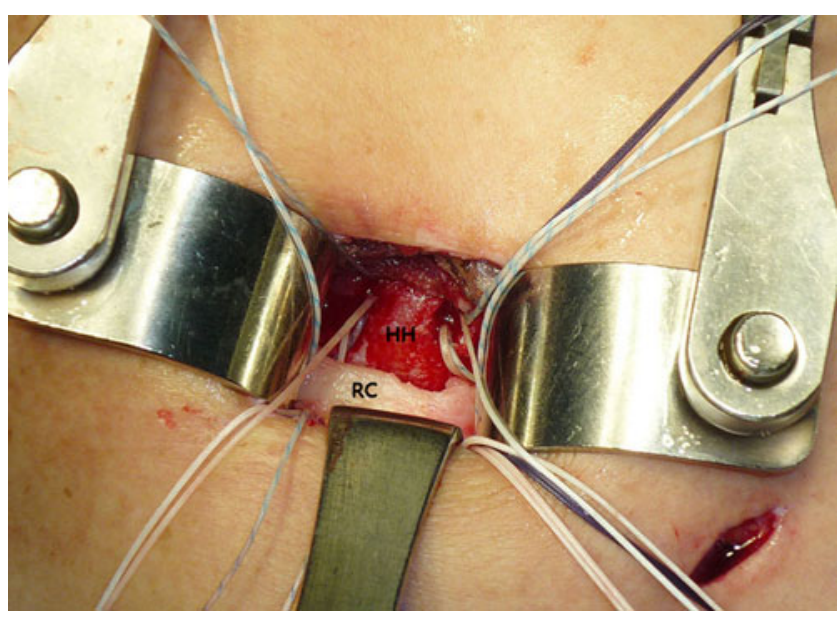

Fig. 2 Placement of a deltoid retractor to allow direct visualisation of the torn rotator cuff $(\mathrm{RC})$ and humeral head $(\mathrm{HH})$

\section{Clinical assessment}

We evaluated preoperative and final follow-up visual analogue scale (VAS) and activities of daily living (ADL) scores for each patient. Clinical outcomes for all patients were evaluated using the American Shoulder and Elbow Surgery (ASES) scoring system. All patients completed the ASES subjective questionnaire preoperatively and at final follow-up. The shoulder index, as calculated by the ASES scoring system, was used to obtain overall results: excellent $\geq 90$; good 80-89; fair, 70-79; poor $<70$.

\section{Statistical analysis}

Statistical analysis was performed using SPSS software (version 15.0E; SPSS Inc., Chicago, IL, USA). To examine

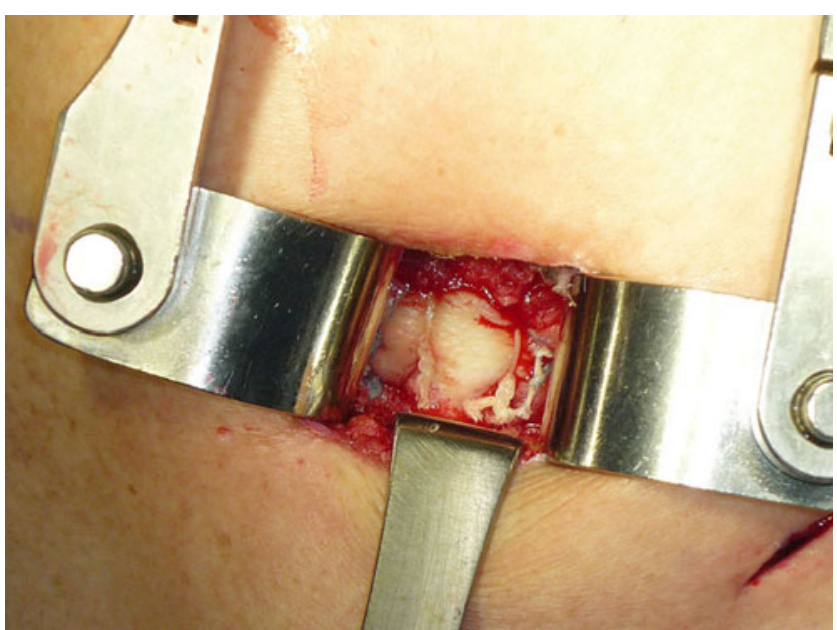

Fig. 3 The torn tendon was repaired anatomically with the doublerow technique using suture anchors

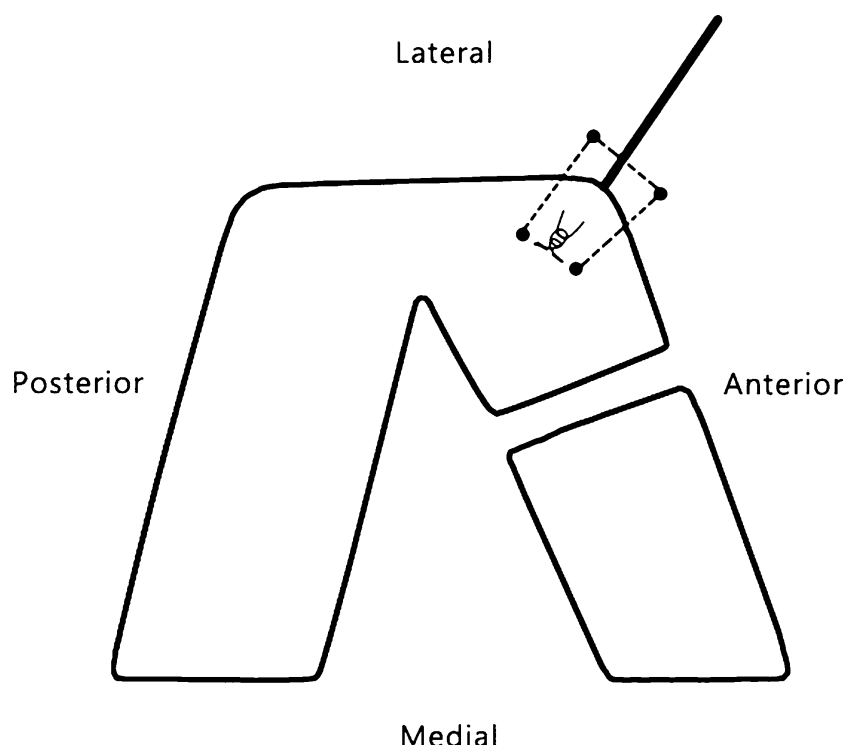

Fig. 4 Additional suturing within the bone tunnel to prevent avulsion of the deltoid from the acromion

baseline characteristics, we analysed frequency and descriptive statistics. To determine the correlation between clinical outcomes and various parameters such as age, sex, affected side, symptom duration, preoperative stiffness and tear size, we used the independent $t$ test, Pearson correlation analysis and one-way analysis of variance (ANOVA). Statistical significance was set at $p<0.05$.

\section{Results}

Rotator cuff tear sizes confirmed intraoperatively were eight (6.3\%) partial thickness, 26 (20.3\%) small, 40 (31.3\%) medium, 39 (30.5\%) large and 15 (11.7\%) massive. At the time of repair, nine $(7.0 \%)$ patients also had a capsular release, $12(9.4 \%)$ a biceps tenotomy, five (3.9\%) a biceps tenodesis and one $(0.8 \%)$ a distal clavicle resection. Analyses of preoperative ASES scores and various parameters showed statistically significant lower preoperative ASES scores in women $(P=0.015)$ and patients with preoperative stiffness $(P=0.003)$. There were no statistically significant differences in preoperative ASES scores by age, affected side, symptom duration or tear size () (Table 1).

The average VAS scores improved from 6.6 (range three to ten) preoperatively to 1.2 (range zero to six) at final follow-up $(P<0.0001)$. The average ADL scores improved from 12.0 (range 2-28) preoperatively to 26.6 (range nine to 30) at final follow-up $(P<0.0001)$. The average ASES scores improved from 36.6 (range three to 77 ) preoperatively to 88.2 (range 44.9-99.8) at final follow-up $(P<0.0001)$ (Table 2). There were 71 (55.5\%) excellent, 39 (30.5\%) 
Table 1 Statistical correlations between variables and preoperative American Shoulder and Elbow Surgeon (ASES) scores

n.s. not significant

\begin{tabular}{llccc}
\hline Variables & & Number & Mean preoperative ASES score & $P$ value \\
\hline Sex & Male & 80 & $39.2 \pm 15.5$ & $0.015^{*}$ \\
Affected side & Female & 40 & $32.4 \pm 14.5$ & \\
& Dominant & 90 & $88.7 \pm 10.2$ & n.s. \\
Preoperative Stiffness & Nondominant & 38 & $89.4 \pm 12.6$ & \multirow{2}{*}{$0.003^{*}$} \\
& Stiff & 110 & $38.3 \pm 15.5$ & n.s. \\
Tear size & Not stiff & 18 & $26.7 \pm 10.6$ & \\
& Partial & 8 & $30.4 \pm 15.8$ & \\
& Small & 26 & $35.1 \pm 14.9$ & \\
& Medium & 40 & $33.8 \pm 16.9$ & n.s. \\
Age & Large & 39 & $39.0 \pm 14.3$ & n.s. \\
Symptom duration & Massive & 15 & $36.7 \pm 15.4$ & \\
\hline
\end{tabular}

good, ten $(7.8 \%)$ fair and eight $(6.3 \%)$ poor results. There were no statistically significant differences between final follow-up ASES scores and age, affected side, symptom duration, preoperative stiffness or tear size, but men showed significantly higher final ASES scores than women $P=0.014$ ) (Table 3). Postoperative complications included stiff shoulder in seven $(5.5 \%)$ patients, reoperation because of retear in two $(1.6 \%)$ and postoperative infection in one $(0.8 \%)$.

\section{Discussion}

The most important finding of this study was that our technique provided better visualisation for rotator cuff tear of all sizes and satisfactory clinical outcomes. Mini-open rotator cuff repair involves arthroscopic subacromial decompression followed by a deltoid-splitting approach for the repair. It is a well-established, excellent technique for treating full-thickness tears and offers many advantages of either open or arthroscopic repair while minimising the disadvantages. Mini-open repair is still commonly used and a promising technique that can reduce postoperative pain and deltoid morbidity. Also, clinical results are well documented and compare favourably with those for open or allarthroscopic technique [10, 11, 14, 18]. Recent studies comparing mini-open repair with all-arthroscopic repair

Table 2 Visual analogue score (VAS), activities of daily living (ADL and American Shoulder and Elbow Surgeon (ASES) scores

\begin{tabular}{lccc}
\hline & Preoperative & Final & $P$ value \\
\hline VAS & $6.6 \pm 1.9$ & $1.2 \pm 1.2$ & $<.0001^{*}$ \\
ADL & $12.0 \pm 5.7$ & $26.6 \pm 3.4$ & $<.0001^{*}$ \\
ASES & $36.7 \pm 15.4$ & $88.2 \pm 10.9$ & $<.0001^{*}$ \\
\hline
\end{tabular}

show no statistically significant difference in functional outcome between the two techniques [1, 2, 5-9, 12, 13, 15]. However, most studies comparing mini-open repair with all-arthroscopic repair in patients with full-thickness tears are neither randomised controlled trials nor prospective cohort studies.

Mini-open techniques include lateral portal-extension approach, anterior portal-extension approach and deltoid-on approach $[14,17,18,20,22,23]$. The lateral portalextension approach is most commonly used, involving lateral deltoid splitting, and has proven satisfactory in longterm clinical results $[7,12,14,23]$. However, it still leads to significant deltoid and axillary nerve injury from excessive traction during attempts to repair large or massive tears and limits visualisation of medially retracted cuff tears and subscapularis tears. The anterior portal extension approach provides good visualisation and avoids axillary nerve injury, but it is difficult to assess posterior cuff tear [11, 19, 20]. Matsen and Lippitt [21] described mini-open repair with a deltoid-on approach carried out through a 3- to 4-cm split in the most prominent anterolateral raphe of the deltoid muscle near its origin from the acromion. Several authors reported that during mini-open repair, blunt dissection between the anterior and lateral raphe of the deltoid muscle allows rotator cuff exposure without deltoid detachment or injury. [15].

Our anterolateral mini-open technique using a modified deltoid-on approach, representing a smaller version of a standard open repair, has several advantages. It allows direct access to the anterior supraspinatus, which is the most common site of rotator cuff tears. In smaller tears, the cuff will be clearly visible in the deltoid split. In larger tears, the arm can be rotated into extension and internally to allow better visualisation of the posterior aspect of the rotator cuff. Flexion and external rotation allows excellent visualisation of the biceps groove and subscapularis. Also, 
Table 3 Statistical correlations between variables and final American Shoulder and Elbow Surgeon (ASES) scores

n.s. not significant

\begin{tabular}{lllll}
\hline Variables & & & & \\
\hline Sex & Nale & 80 & $90.0 \pm 10.1$ & \multirow{2}{*}{$0.014^{*}$} \\
Affected side & Female & 40 & $85.1 \pm 11.7$ & \\
& Dominant & 90 & $88.7 \pm 10.2$ & n.s. \\
Preoperative Stiffness & Nondominant & 38 & $89.4 \pm 12.6$ & n.s. \\
& Stiff & 110 & $88.3 \pm 11.4$ & n.s. \\
Tear size & Not stiff & 18 & $87.6 \pm 7.28$ & \\
& Partial & 8 & $80.5 \pm 9.4$ & \\
& Small & 26 & $88.2 \pm 12.0$ & \\
& Medium & 40 & $89.4 \pm 10.3$ & n.s. \\
Age & Large & 39 & $90.1 \pm 10.7$ & n.s. \\
Symptom duration & Massive & 15 & $84.2 \pm 10.7$ & \\
\hline
\end{tabular}

our technique makes it possible for less experienced surgeons to confirm adequacy of subacromial decompression by direct digital palpation and to perform biceps tenodesis easily.

Previous research has been controversial regarding the effect of tear size and age on outcome after rotator cuff repair, with some studies reporting that these factors influenced outcome and others reporting the opposite [6, 17, 22-24]. Romeo et al. [24] reported that tears $\geq 5 \mathrm{~cm}^{2}$ were associated with a poorer outcome. However, Baysal et al. [22] prospectively reviewed 84 patients with tears of all sizes, including 17 with large or massive tears, who underwent mini-open repair, and reported a statistically significant improvement in shoulder scores and range of motion. They found no difference in outcome between different tear sizes. Subsequent reports demonstrated that the mini-open technique can be used effectively for even large and massive tears [19, 20, 23]. In our study, patients experienced significant decreases in pain and had significant improvement of pain and ASES scores after surgery. Of 128 patients, $110(86.0 \%)$ had satisfactory clinical outcomes. It is especially noteworthy that $47(87.0 \%)$ of 54 patients with large or massive tears had satisfactory clinical outcomes. Our data are consistent with those of previous studies that obtained $>80 \%$ good or excellent clinical results with the mini-open technique [3]. Our subgroup analysis showed no statistically significant difference between final follow-up ASES scores and age, affected side, symptom duration, preoperative stiffness or tear size, but men had significantly higher final ASES scores than women. We found that our technique provided both adequate exposure for repair of tears of all sizes and satisfactory clinical outcomes for patients with large to massive tear.

One disadvantage of the mini-open repair is that it can produce shoulder stiffness after surgery. Incidence ranges between $11 \%$ and $20 \%$ have been reported $[1,4,16]$. However, Youm et al. [25] compared outcomes of allarthroscopic repair versus mini-open repair and found no evidence of stiffness in patients treated with the mini-open technique. Although several studies have shown increased range of motion for the all-arthroscopic repair in the early postoperative period, Verma et al. [2] reported no difference after all-arthroscopic versus mini-open repair at the twoyear follow-up. In our study, seven patients (5.5\%) had postoperative shoulder stiffness; six of them had small or medium tears and one had a massive tear. These results are similar to those reported for all-arthroscopic repair. To prevent avulsion of the deltoid from the acromion, additional suturing with bone tunnel was performed using no. 1 absorbable suture with cutting needle. We believe that our technique can decrease the risk of deltoid avulsion because it allows excellent visualisation of even larger retracted tears and prevents deltoid injury with the use of additional sutures.

The limitations of our study were that it was retrospective in nature and that it did not compare our outcome with a cohort that underwent an open or all-arthroscopic technique. Furthermore, we did not assess the structural integrity of repaired rotator cuffs at follow-up evaluations. There is a need for a prospective, randomised clinical trial with prespecified hypothesis and confirmatory postoperative imaging studies to evaluate outcomes of rotator cuff repair with either a mini-open or an allarthroscopic technique.

\section{Conclusion}

Anterolateral approach for mini-open rotator cuff repair produces satisfactory results. It may also provide better visualisation for rotator cuff tears of all sizes. 


\section{References}

1. Morse K, Davis AD, Afra R, Kaye EK, Schepsis A, Voloshin I (2008) Arthroscopic versus mini-open rotator cuff repair: a comprehensive review and meta-analysis. Am J Sports Med 36:1824-1828. doi:10.1177/0363546508322903

2. Verma NN, Dunn W, Adler RS, Cordasco FA, Allen A, MacGillivray $\mathrm{J}$ et al (2006) All-arthroscopic versus mini-open rotator cuff repair: a retrospective review with minimum 2-year follow-up. Arthroscopy 22:587-594. doi:10.1016/j.arthro.2006.01.019

3. Yamaguchi K (2001) Mini-open rotator cuff repair: an updated perspective. Instr Course Lect 50:53-61

4. Yamaguchi K, Ball CM, Galatz LM (2001) Arthroscopic rotator cuff repair; transition from mini-open to all-arthroscopic. Clin Orthop Relat Res 390:83-94

5. Duquin TR, Buyea C, Bisson LJ (2010) Which method of rotator cuff repair leads to the highest rate of structural healing? A systematic review. Am J Sports Med 38:835-841. doi:10.1177/ 0363546509359679

6. Nho SJ, Shindle MK, Sherman SL, Freedman KB, Lyman S, MacGillivray JD (2007) Systematic review of arthroscopic rotator cuff repair and mini-open rotator cuff repair. J Bone Joint Surg Am 89:127-136. doi:10.2106/JBJS.G.00583

7. Osti L, Papalia R, Paganelli M, Denaro E, Maffulli N (2010) Arthroscopic vs mini-open rotator cuff repair. A quality of life impairment study. Int Orthop 34:389-394. doi:10.1007/s00264009-0796-Z

8. Razmjou H (2009) Evaluating equivalency of treatment effectiveness: the example of arthroscopic and mini-open rotator cuff repairs. Hand Clin 25:67-70. doi:10.1016/j.hcl.2008.10.002

9. Churchill RS, Ghorai JK (2010) Total cost and operating room time comparison of rotator cuff repair techniques at low, intermediate, and high volume centers: mini-open versus allarthroscopic. J Shoulder Elbow Surg 19:716-721. doi:10.1016/j. jse.2009.10.011

10. Hata Y, Saitoh S, Murakami N, Kobayashi H, Takaoka K (2004) Atrophy of the deltoid muscle following rotator cuff surgery. $\mathrm{J}$ Bone Joint Surg Am 86:1414-1419

11. Hata Y, Saitoh S, Murakami N, Seki H, Nakatsuchi Y, Takaoka K (2001) A less invasive surgery for rotator cuff tear: mini-open repair. J Shoulder Elbow Surg 10:11-16. doi:10.1067/mse.2001.110514

12. Kang L, Henn RF, Tashjian RZ, Green A (2007) Early outcome of arthroscopic rotator cuff repair: a matched comparison with miniopen rotator cuff repair. Arthroscopy 23:573-582. doi:10.1016/j. arthro.2007.01.011

13. Kim SH, Ha KI, Park JH, Kang JS, Oh SK, Oh I (2003) Arthroscopic versus mini-open salvage repair of the rotator cuff tear: outcome analysis at 2 to 6 years' follow-up. Arthroscopy 19:746-754. doi:10.1016/S0749-8063(03)00395-5

14. Mohtadi NG, Hollinshead RM, Sasyniuk TM, Fletcher JA, Chan DS, Li FX (2008) A randomized clinical trial comparing open to arthroscopic acromioplasty with mini-open rotator cuff repair for full-thickness rotator cuff tears: disease-specific quality of life outcome at an average 2-year follow-up. Am J Sports Med 36:1043-1051. doi:10.1177/0363546508314409

15. Pearsall AW 4th, Ibrahim KA, Madanagopal SG (2007) The results of arthroscopic versus mini-open repair for rotator cuff tears at mid-term follow-up. J Orthop Surg Res 1:24. doi:10.1186/ 1749-799X-2-24

16. Williams G, Iannotti J, Lichetti W, Ferron A (1998) Mini versus open repair of isolated supraspinatus tendon tears. J Shoulder Elbow Surg 7:310

17. MacDougal GA, Todhunter CR (2010) Delamination tearing of the rotator cuff: prospective analysis of the influence of delamination tearing on the outcome of arthroscopically assisted mini open rotator cuff repair. J Shoulder Elbow Surg 19:10631069. doi:10.1016/j.jse.2009.12.020

18. Park JY, Levine WN, Marra G, Pollock RG, Flatow EL, Bigliani LU (2000) Portal-extension approach for the repair of small and medium rotator cuff tears. Am J Sports Med 28:312-316

19. Chun JM, Kim SY, Kim JH (2008) Arthroscopically assisted minideltopectoral rotator cuff repair. Orthopedics 31:74

20. Duralde XA, Greene RT (2008) Mini-open rotator cuff repair via an anterosuperior approach. J Shoulder Elbow Surg 17:715-721. doi:10.1016/j.jse.2008.01.142

21. Matsen FA 3rd, Lippitt SB (2004) Procedure: rotator cuff repair. In: Matsen FA 3rd, Lippitt SB, DeBartolo SE (eds) Shoulder surgery: principle and procedures. WB Saunders, Philadelphia, pp 347-377

22. Baysal D, Balyk R, Otto D, Luciak-Corea C, Beaupre L (2005) Functional outcome and health-related quality of life after surgical repair of full-thickness rotator cuff tear using a miniopen technique. Am J Sports Med 33:1346-1355. doi:10.1177/ 0363546505275130

23. Hanusch BC, Goodchild L, Finn P, Rangan A (2009) Large and massive tears of the rotator cuff: functional outcome and integrity of the repair after a mini-open procedure. J Bone Joint Surg Br 91:201-205. doi:10.1302/0301-620X.91B2.21286

24. Romeo AA, Hang DW, Bach BR Jr, Shott S (1999) Repair of full thickness rotator cuff tears. Gender, age, and other factors affecting outcome. Clin Orthop Relat Res 367:243-255

25. Youm T, Murray DH, Kubiak EN, Rokito AS, Zuckerman JD (2005) Arthroscopic versus mini-open rotator cuff repair: a comparison of clinical outcomes and patient satisfaction. J Shoulder Elbow Surg 14:455-459. doi:10.1016/j.jse.2005.02.002 